\title{
QUARANTINE LENGTH AND SURVIVAL OF TRANSLOCATED EUROPEAN WILD RABBITS
}

\author{
CARLOS CALVETE, ${ }^{1,2}$ Instituto de Investigación de Recursos Cinegéticos, (IREC) Ronda de Toledo s/n. 13005, Ciudad Real, \\ Spain \\ ELENA ANGULO, ${ }^{3}$ Estación Biológica de Doñana (CSIC), Apdo. 1056, E-41080, Sevilla, Spain \\ ROSA ESTRADA, Instituto de Investigación de Recursos Cinegéticos, (IREC) Ronda de Toledo s/n. 13005, Ciudad Real, Spain \\ SACRAMENTO MORENO, Estación Biológica de Doñana (CSIC), Apdo. 1056, E-41080, Sevilla, Spain \\ RAFAEL VILLAFUERTE, Instituto de Investigación de Recursos Cinegéticos, (IREC) Ronda de Toledo s/n. 13005, Ciudad Real, \\ Spain
}

\begin{abstract}
European wild rabbits (Oryctolagus cuniculus) are frequently translocated for hunting and conservation purposes. Quarantining these animals prior to release reduces the risk of releasing rabbits incubating field infections of myxomatosis or viral haemorrhagic disease (RHD), and it provides a way to vaccinate these animals against both diseases. However the optimal quarantine period needed to achieve these goals is not known. We therefore assessed the effects of quarantine lengths (2, 4, 6, 8 weeks) on rabbit biochemical parameters, immunity induced by vaccination against myxomatosis and RHD, and survival of translocated rabbits. We found that levels of total bilirubin, urea nitrogen, aspartate aminotransferase (AST), and alanine aminotransferase (ALT) enzymatic activities were significantly $(P<0.05)$ decreased during quarantine, independent of quarantine length, whereas hematocrit levels increased significantly. All pregnant female rabbits aborted or lost litters during quarantine. Seroconversion against myxomatosis and RHD following vaccination was not related to any biochemical parameter at vaccination time, but the proportion of seronegative rabbits that seroconverted was moderate. The heterophil/lymphocyte ratio, conjugated to unconjugated bilirubin ratio, and the serum AST and creatinine levels we measured after capture and transport were directly related to mortality through the quarantine period, whereas we found total serum protein level was negatively related to mortality. Mortality after release was positively related to urea nitrogen concentration and negatively related to hematocrit and the albumin/globulin ratio, but it was independent of quarantine length. Based on our findings, rabbit translocation programs that include a quarantine period could be improved by decreasing the acute stress induced by capture and handling prior to quarantine; facilitating a more rapid access to high quality feed during quarantine; and improving the vaccination protocol. In addition, the release from quarantine of rabbits should be determined by their physical condition, not merely by elapsed time in quarantine.
\end{abstract}

Key words: body condition, capture, European wild rabbit, mortality, Oryctolagus cuniculus, quarantine, radiotelemetry, Spain, translocation, vaccination.

Translocation, or the deliberate and mediated movement of wild individuals or populations from 1 part of their range to another (IUCN 1996, Griffith et al. 1989), is an important wildlife management tool. While many factors are involved in translocation success, the possible transmission of new disease agents into the release area is of primary importance because these diseases may have a major impact on native species (Viggers et al. 1993, Wolf et al. 1996). One of the steps taken to minimize the risk of disease transmission during translocations is disease monitoring in the source population and in the native population that in-

\footnotetext{
${ }^{1}$ Present address: Pza. Serrano Berges 13, $8^{\circ}$ D. 50016 Zaragoza, Spain.

2 Corresponding author e-mail: vetecal2003@jazzfree.com

${ }^{3}$ Instituto de Investigación de Recursos Cinegéticos, (IREC) Ronda de Toledo s/n. 13005, Ciudad Real, Spain
}

habits the release area. The main veterinary tools used to reduce the risk of importing new pathological agents are sanitary control, which consists primarily of quarantining animals for a specific period of time prior to translocation, and treating these animals prophylactically against diseases known to be endemic in the release area (e.g., by vaccination; Jacobson 1993, Spalding and Forrester 1993, Viggers et al. 1993). The quarantine period is dependent on the maximum incubation period of diseases known to affect the animal (Woodford and Kock 1991), but it may be extended when animals are incubating a field infection or are developing immunity induced by vaccination. However, quarantine can place a high level of stress on animals caught in the wild, and this level of stress is dependent on the species and individual characteristics of the animals (Woodford and Rossiter 1993). In designing translocation programs, veterinarians and biologists must take into account 
the trade-off between minimizing disease risks in the native and translocated species and the effects of the quarantine period on the health, behavior, and survival of the translocated species.

In Spain, translocation programs for the European wild rabbit are designed to increase populations for hunting and conservation (Calvete et al. 1997, Angulo 2003). The European wild rabbit is considered the most important small game species in sport hunting and is the main prey species for most vertebrate predators (Angulo 2003). Several predators that are threatened with extinction (e.g., Iberian lynx [Lynx pardina], imperial eagle [Aquila adalberti]), are dependent on abundant rabbit populations (Delibes and Hiraldo 1981). The arrival of myxomatosis in Spain in the 1950s (Muñoz 1960) and viral haemorrhagic disease (RHD) in 1989 (Argüello et al. 1988), however, led to substantial reductions in rabbit population density (Angulo and Cooke 2002). Attempts to boost rabbit populations by translocations have increased, and the total number of translocated wild rabbits has been considerable (e.g., about 4,300 rabbits were released in Doñana National Park from 1993 to 1995 with negligible success [R. Villafuerte, unpublished data]).

Myxomatosis and RHD have become endemic diseases, and periodic outbreaks have a substantial impact on wild rabbit populations (Calvete et al. 2002) resulting in an ongoing need for translocation programs. During large-scale, long-term translocation programs, however, translocated rabbits are taken simultaneously from several wild populations, making the updated sanitary monitoring of these source populations unreliable and increasing the probability of capturing animals incubating myxomatosis and RHD. Thus, a quarantine period is likely the most powerful sanitary tool to avoid the release of animals incubating myxomatosis and RHD among native rabbits; a quarantine period can also be used to immunize translocated rabbits by vaccination against both diseases prior to release.

The utility of quarantine was tested by Calvete et al. (1997), who enhanced survival of batches of translocated rabbits by controlling clearly diseased or injured animals. The incubation period of myxomatosis and the time following vaccination necessary to develop immunity against RHD and myxomatosis in domestic rabbits indicate that the quarantine period should last at least 12-18 days (Argüello 1986, 1991). However, rabbits may be quarantined for several weeks or months when animals that will be translocated are incubating myx- omatosis or when there is need to conform to a planned release schedule. To date, the effects of quarantine length on the rabbits' physiological responses and survival after release and on the efficacy of vaccination have not been tested.

As part of its program to conserve several predator species, the Department of Natural and $\mathrm{Na}$ tional Parks of Doñana, in southwestern Spain, conducted wild rabbit translocation programs for several years, and the rabbits were quarantined prior to release. To determine the optimal quarantine period, we evaluated the effects of quarantine length on the physiological status of rabbits, on the efficacy of immunization induced by vaccination against myxomatosis and RHD, and on short-term survival after release.

\section{METHODS}

\section{Capture and Transportation}

During April 2001, trappers captured adult wild rabbits from several wild populations located around the area of Doñana; we used rabbits from this area to ensure that all captured rabbits had a high genetic similarity to Doñana rabbits (Branco 1995, Ferrand 1995). Trappers captured rabbits by ferreting and confined them for 6-40 hr in small yards with drinking water and straw as bedding before transportation. Since individuals from different populations were mixed prior to transportation to quarantine facilities, we were unable to document the exact origin of each rabbit and the exact length of time each was confined prior to being received by the quarantine facilities.

Prior to transport, we sprayed rabbits with diluted cipermethrine insecticide to eliminate ectoparasites. Rabbits were confined in plastic boxes $(70 \times 50 \times 16 \mathrm{~cm}), 4-6$ per box, and transported by car to the quarantine facilities, a trip that took 1-2 hr.

\section{Quarantine}

Upon arrival at quarantine facilities, we weighed and identified the sex of all rabbits and gave them numbered metallic ear tags. We tested pregnancy in females by palpation. We initially housed 67 rabbits (39 females, 28 males) in quarantine facilities, and we confirmed pregnancy in 24 (61.5\%) females. We incised the auricular marginal vein to obtain a 3-ml blood sample without anticoagulant, 2 micro-capillary tubes with $\mathrm{K}_{3}$ EDTA anticoagulant, and 2 blood smears from each rabbit. We subdivided serum removed from blood without anticoagulant into aliquots. Serum was frozen at 
$\leq 20^{\circ} \mathrm{C}$ for 1 week and then kept at $\geq 80^{\circ} \mathrm{C}$ until analyzed. We subcutaneously vaccinated all rabbits against RHD (Cylap-VHD, Sobrino-Cyanamid Laboratory, Madrid, Spain) and in the ear skin with myxomatosis commercial vaccine against Sanarelli virus (Pox-Lap, Ovejero Laboratory, León, Spain) using a Dermoject injector. Established quarantine protocol also included subcutaneous inoculations against nematodes with levamisol (Nemisol, Ovejero Lab., León, Spain), cestodes with praziquantel (Droncit, Bayer Hispania Laboratory, Barcelona, Spain), and trematodes by the oral administration of triclabendazole (Fasinex, Novartis Farmaceútica Laboratory, Barcelona, Spain). We administered all products at doses recommended for domestic rabbits. We prevented coccidiosis cases during quarantine by administering robenidine coccidiostatic with the commercial rabbit pellet $(60 \mathrm{mg} / \mathrm{kg})$.

Following inoculations, we housed rabbits individually in flat-deck type cages commonly used for commercial breeding of domestic rabbits. We provided lucerne hay and straw in each cage as bedding and nesting material. All rabbits had free access to water and to food supplies of lucerne hay supplemented with commercial rabbit pellets (16\% crude protein). To avoid pathological enteric processes caused by rapid change in diet, we progressively introduced rabbits to commercial pellets, with unlimited access 9-11 days after the start of quarantine. On the day prior to release, we weighed rabbits and obtained blood samples following the same protocol as above. We inoculated rabbits with a booster injection against RHD and tagged them with a radiocollar weighing approximately $20 \mathrm{~g}$ and containing an activity sensor (AVM Instrument Company, California, USA). To avoid variations due to adaptation to repeated handling, we handled each rabbit only twice to draw blood. We took all blood samples from 1000 to $1300 \mathrm{hr}$.

\section{Blood Analyses}

We determined the biochemical parameters spectrophotometrically from serum samples; these included total bilirubin, conjugated and unconjugated bilirubin, aspartate aminotransferase (AST) and alanine aminotransferase (ALT), urea nitrogen, creatinine, and total protein. We determined concentrations of the serum proteins albumin, total globulins, and $\gamma$-globulins electrophoretically using cellulose acetate. We used a hematocrit centrifuge to determine hematocrit. We performed differential leukocyte counts on blood smears stained with a commercial stain (Quick panoptic, QCA Lab., Madrid, Spain). We observed cells under $\times 1,000$ magnification; for each sample, we classified 100 leukocytes and calculated the heterophil/lymphocyte ratio.

To evaluate the efficacy of vaccination, we determined serum antibody concentrations against RHD and myxomatosis using commercial ELISA kits (Ingezim rabbit, Ingenasa Laboratory, Madrid, Spain, for RHD; and CIVTest cuni, Hipra Laboratory, Gerona, Spain, for myxomatosis). We expressed antibody concentration as Relative Index of Immunity (RI), in which the optical density (OD) of each sample was related to the ODs of positive and negative controls. Relative Index of Immunity values ranged from 1 to 10 , and we scored sera with $\mathrm{RI} \geq 2$ as positive.

\section{Release, Monitoring, and Determination of Survival}

To assess the effects of quarantine length on survival and biochemical parameters, we released randomly selected rabbits in groups of 11 ( 7 females, 4 males), 20 (10 females, 10 males), 15 (10 females, 5 males), and 13 (8 females, 5 males) animals after $2,4,6$, and 8 weeks of quarantine, respectively. We randomly subdivided each group of rabbits into 2 subgroups for release at 2 different release areas that were $2 \mathrm{~km}$ from each other in Doñana Biological Reserve in Doñana National Park. At each release site, we built 6 artificial warrens 25-50-m apart. Each warren was $3 \times 3 \mathrm{~m}$ in size, had 2 entrances, and was constructed of wood planks and tree branches buried $1 \mathrm{~m}$ under the soil surface. To reduce initial mortality and dispersal of rabbits, we enclosed each warren within an $80-\mathrm{cm}$ high wire mesh fence, creating a fenced area of about 6-8-m diameter. Following their release from the warrens in the morning, we confined rabbits to the fenced areas until the fourth day after release (3 nights), during which time they had unlimited access to water and lucerne hay (Calvete and Estrada 2004). We monitored the enclosures daily during this period to verify that all the radiotagged rabbits remained within them. Subsequently, we removed the fences, and the rabbits were released from the enclosures.

We monitored rabbits for 90 days after release using daily telemetry location during the first 2 hours after dawn and dusk. We carried out radiolocation from fixed antennas on towers $4 \mathrm{~m}$ in height and $500 \mathrm{~m}$ from the release area to avoid disturbing the rabbits. When we did not observe change in an activity sensor we located the rabbit 


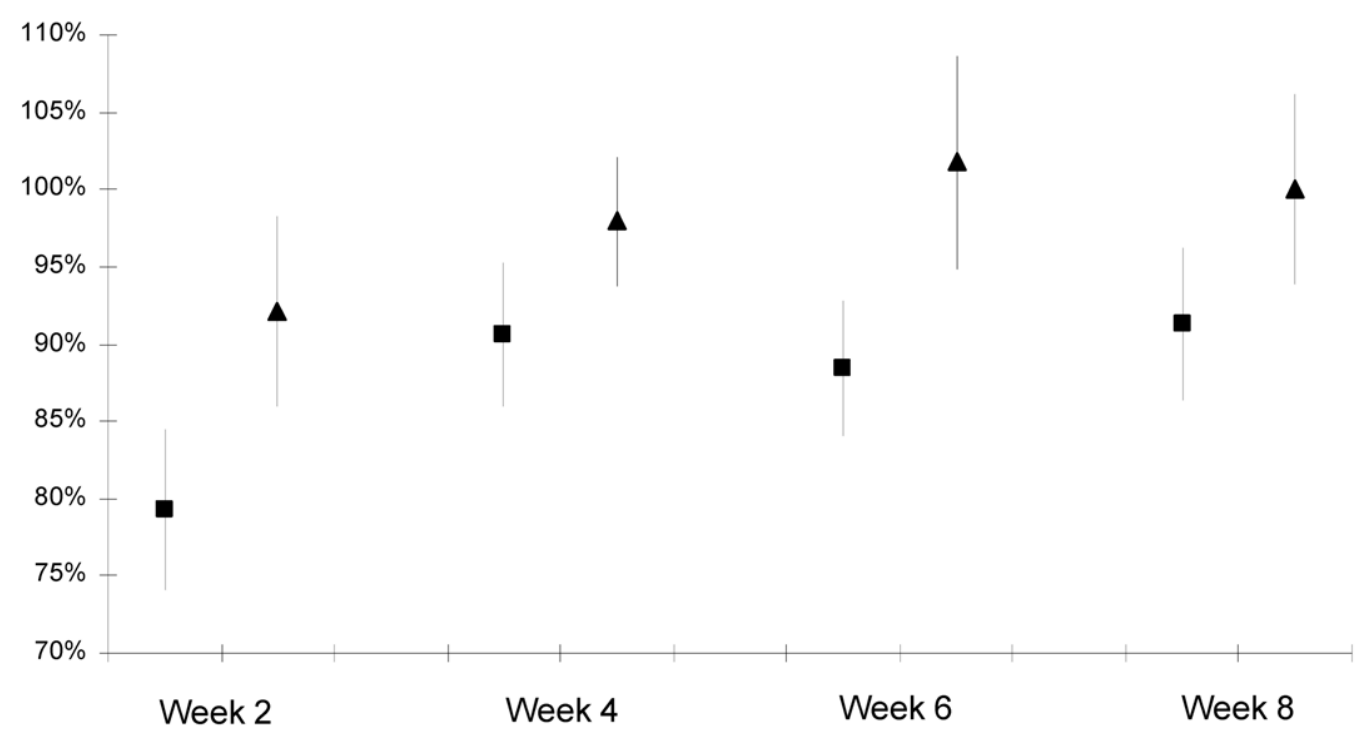

Fig. 1. Variation of body mass of quarantined European wild rabbits at different quarantine lengths expressed as body mass at release relative to body mass at capture. Ratios are expressed as percents. Confidence intervals are set at $95 \%$. All rabbits had $100 \%$ values at week zero. Squares: females, triangles: males.

using a hand-held antenna and physically approached to visually determine if it was alive or dead.

\section{Data Analysis}

We analyzed the effect of quarantine length on body mass by ANOVA, with pregnancy nested within sex and the 4 quarantine length groups as factors. We expressed the dependent variable as the body mass at release relative to the body mass at the start of quarantine.

We assessed the combined variation of all biochemical parameters throughout the quarantine by MANOVA for repeated measures with 2 levels (quarantine factor); and we used pregnancy nested within sex and quarantine length as the between factor design. We determined the variation of each individual parameter by subsequent ANOVAs for repeated measures, with 2 levels for each factor (i.e., at the start and end of quarantine). In addition, we determined the absolute variation of RI through quarantine by ANOVA for repeated measures. We used pregnancy nested within sex and quarantine length as the between factor design and RI at the start and end of quarantine as the within factor design, with 2 levels.

Since rabbits had high prevalence of natural antibodies against RHD and myxomatosis at the start of quarantine, we determined the dynamic of immunity induced by vaccination and the possible associ- ation between immunity and the physiological status of rabbits; as a first step, we used a simple linear regression with no intercept. In our analysis the dependent and independent variables were RI values at the end and at the start of quarantine, respectively. We used standardized residuals of this regression as dependent variables in a subsequent multiple regression analysis; we included the following in the initial model as independent variables: sex and gestation (as dummy variables), biochemical parameters, duration of quarantine (in days) and RI at the start of quarantine. We used a backward elimination procedure to obtain the final model.

We employed Cox's proportional hazard regression model for censored data (Therneau and Grambsch 2001) to determine the association between the risk of dying during quarantine and biochemical values at the start of quarantine. We included sex and gestation in the initial model as dummy independent variables. We obtained the final model by a backward selection procedure based on the likelihood ratio test (LRT).

We used a second Cox's proportional hazard regression model to determine the association between the risk of dying after release and the biochemical parameters, and the association between the risk of dying and the time the rabbits remained in quarantine. We included the time in quarantine in the initial model as a categorical variable with 4 levels (2, 4, 6, 8 weeks), with 8 weeks being the con- 
trol level. We regarded sex, gestation, and release area as independent variables. We obtained the final model following the same elimination procedure as above. We calculated cumulative survival rates by the Kaplan-Meier product-limit method. Statistically significant differences were established at $P$ values < 0.05 .

\section{RESULTS}

Variation of body mass during quarantine followed a similar pattern for both sexes (Fig. 1). Upon being quarantined, rabbits lost body mass; we observed the lowest mean body mass after 2 weeks of quarantine. Body mass increased between weeks 2 and 4 and was stable over the remaining 4 weeks of quarantine. For male rabbits, the stable body mass values were close to those at the start of quarantine. For female rabbits, body mass values stabilized at lower levels than at the start of quarantine, since all the pregnant females (24/39 or $61.5 \%$ ) aborted or produced litters within the first 5 days of quarantine, probably due to stress. All the litters died a few hours after birth as they were abandoned or killed by the female. An ANOVA model fitted to variation of body mass during quarantine showed statistically significant differences by sex $\left(F_{1,55}=24.39, P<0.001\right)$ and by quarantine length factor $\left(F_{3,55}=5.06, P=0.004\right)$ but not by pregnancy. It is likely that females at the start of gestation were not detected by palpation and that aborted fetuses were not found or were reabsorbed by females. Post-hoc comparisons performed with an LSD Fisher test did not show significant differences between groups for males. However, the body mass ratio of females after 2 weeks of quarantine was significantly lower than after $4(P=0.003), 6(P=0.013)$, or 8 weeks $(P=0.002)$.

Multivariate repeated measures model fitted to the biochemical parameters through quarantine showed that quarantine and quarantine length were the primary factors associated with variation in the biochemical parameters (Wilks $=0.40, F_{11,42}$ $=5.63, P<0.001$; Wilks $=0.35, F_{33,124}=1.61, P=$
0.033 , respectively). Neither sex nor pregnancy had statistically significant effects on these biochemical parameters.

Total bilirubin, AST, ALT, and urea nitrogen all decreased significantly $(P<0.05)$ from the start to the end of quarantine (Table 1), and univariate ANOVA tests showed significant differences by quarantine factor for each of these parameters. We also found a significant increase in hematocrit values during quarantine. The remaining parameters showed no significant changes. Interaction between the quarantine repeated measures factor and quarantine length factor was significantly related to variations in urea nitrogen $\left(F_{3,56}=7.3, P\right.$ $<0.001)$ and hematocrit $\left(F_{3,56}=2.98, P=0.040\right)$. Post-hoc comparisons performed with the LSD Fisher test showed that the group of rabbits released after 2 weeks of quarantine exhibited no significant increase in urea nitrogen $(P=0.870)$, while the rabbits released at $4(P<0.001), 6$ ( $P<$ $0.001)$, and 8 weeks $(P=0.014)$ showed significant decreases. The 2-week group also showed no significant decrease $(P=0.452)$ in hematocrit, in contrast to the increases exhibited by the other 3 groups. In this case, post-hoc comparisons were not statistically significant for the groups released at $4(P=0.123), 6(P=0.140)$, and 8 weeks $(P=$ $0.205)$.

Most rabbits had natural antibodies against RHD (83.6\%) and myxomatosis (80.6\%) at the start of quarantine. Immunity induced by vaccination against myxomatosis and RHD was not significantly associated with any biochemical parameter, sex, pregnancy, or quarantine length $(P>$ 


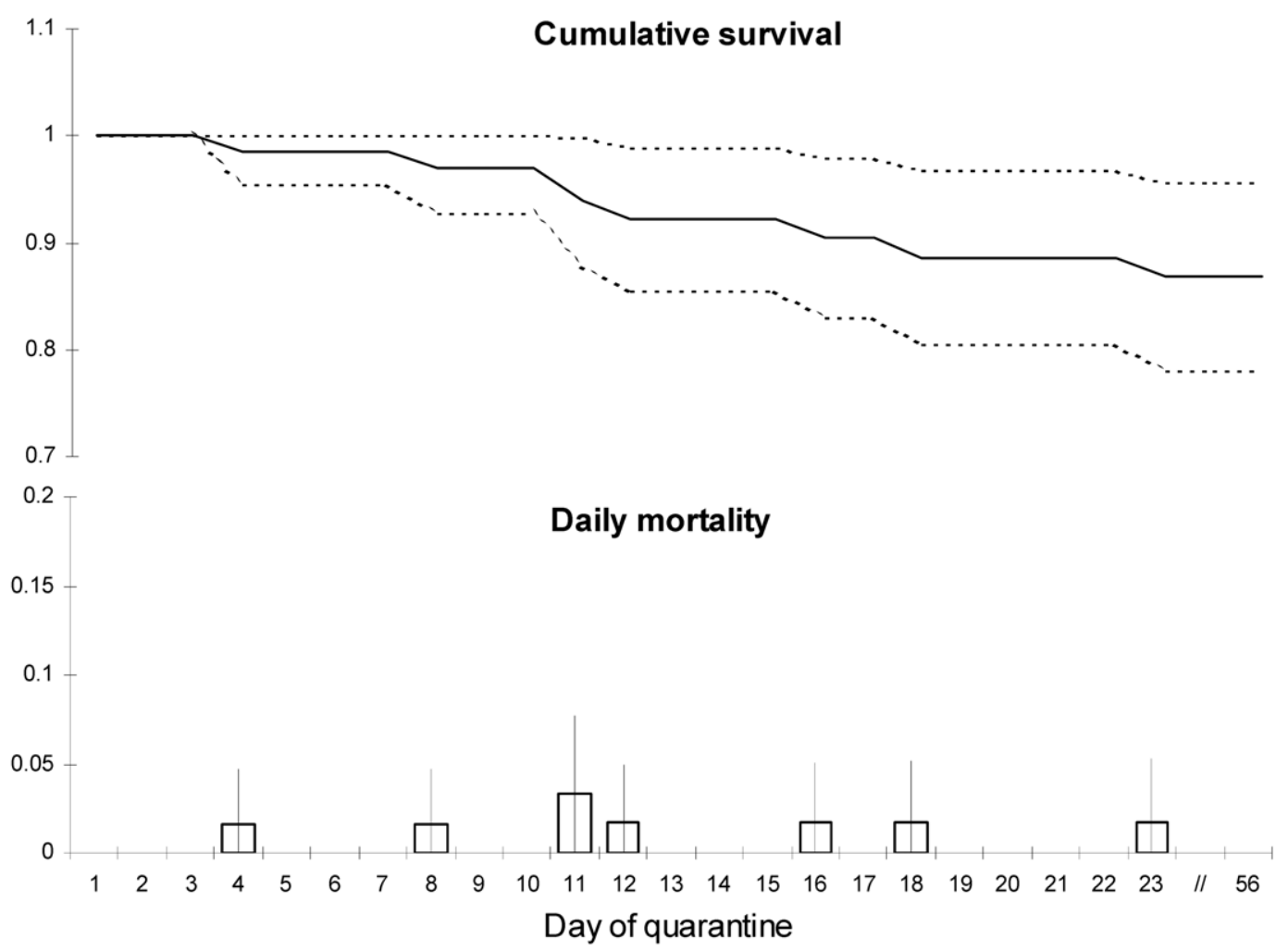

Fig. 2. Daily mortality and cumulative survival rates of European wild rabbits through the quarantine period. Confidence intervals (dotted and vertical lines) are set at 95\%.

0.05). Vaccination against RHD had a significant effect on immunity $\left(F_{1,59}=18.13, P<0.001\right)$, with a mean RI $( \pm$ SE) of $5.70 \pm 0.43$ at the start of quarantine, rising to $6.67 \pm 0.41$ at release. Of the 11 rabbits seronegative at the start of quarantine, however, only 5 (45\%) seroconverted at the time of release. In a similar way, vaccination against myxomatosis also increased immunity significantly $\left(F_{1,59}=35.16, P<0.001\right)$; the mean $( \pm \mathrm{SE}) \mathrm{RI}$ was
$6.09 \pm 0.47$ at the start of quarantine, increasing to $8.53 \pm 0.37$ at release. Of the 13 rabbits seronegative at the start of quarantine, 9 (69\%) seroconverted by the time of release.

Of the 67 rabbits initially housed in quarantine facilities, 4 males and 4 females died during the 56-day quarantine. All deaths occurred between days 4 and 23, resulting in a cumulative survival rate $( \pm \mathrm{SE}$ ) for the whole quarantine period of $86.82 \% \pm 4.37 \%$ (Fig. 2).

Table 2. Biochemical parameters significantly related to risk of dying in translocated European wild rabbits. Cox's regression analysis was utilized to calculate the relationship between biochemical parameters at the start of quarantine and the risk of dying through quarantine and between biochemical parameters at the end of quarantine and the risk of dying after release.

\begin{tabular}{lrrc}
\hline \multicolumn{2}{c}{ Cox's parameter } & SE & P \\
\cline { 1 - 1 } Through quarantine & & & \\
$\quad$ Heterophil/linfocyte ratio & 0.806 & 0.264 & 0.002 \\
Conjugated/unconjugated ratio & 0.013 & 0.006 & 0.032 \\
AST & 0.040 & 0.014 & 0.004 \\
Creatinine & 6.158 & 2.214 & 0.005 \\
Serum proteins & -2.447 & 0.877 & 0.005 \\
After release & & & \\
Albumin/globulins ratio & -6.362 & 2.450 & 0.005 \\
Urea nitrogen & 0.067 & 0.030 & 0.024 \\
Hematocrit & -0.090 & 0.043 & 0.036 \\
\hline
\end{tabular}

Cox's regression model fitted to survival quarantine data $\left(r^{2}=0.35\right.$, LRT $=28.8, \mathrm{df}=5, P<0.001)$ showed that the heterophil/lymphocyte ratin A.ST artivitv rreatinine level, and the conjugated/unconjugated bilirubin ratio at the start of quarantine each showed a significant, positive association with the rate of dying 

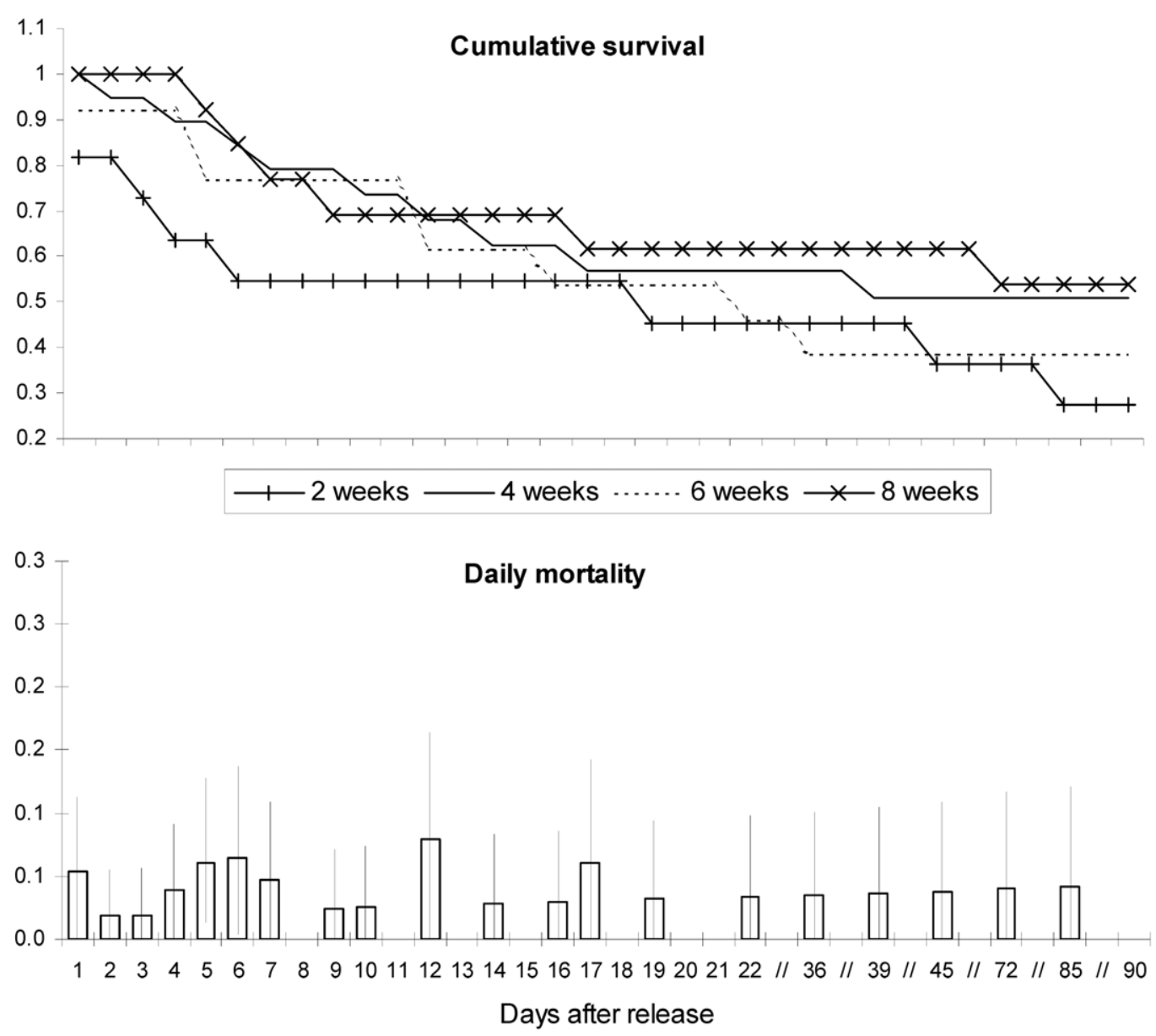

Fig. 3. Survival rates up to 90 days after release of European wild rabbits released after 2, 4, 6 , and 8 weeks of quarantine. Cumulative survival rate for each quarantine length group and daily mortality rate (with $95 \%$ confidence intervals) for all release groups combined.

during quarantine, whereas total serum proteins showed a negative association (Table 2).

After release, the cumulative survival rate $( \pm \mathrm{SE})$ of all rabbits at day 90 of the survey was $43.83 \% \pm$ 6.71\%. Mortality due to translocation was higher during the first days after release, with the main mortality period extending until day 22 (Fig. 3) when the cumulative survival was estimated at $53.15 \% \pm 6.71 \%$. At this time, survival was $45.45 \%$ $\pm 15.01 \%, 56.68 \% \pm 11.6 \%, 46.15 \% \pm 13.83 \%$, and $61.54 \% \pm 13.49 \%$ for the groups released after 2 , 4,6 , and 8 weeks of quarantine, respectively.

Cox's proportional hazard regression model fitted to the survival data up to day 23 after release $\left(r^{2}=0.24\right.$, LRT $\left.=15.5, \mathrm{df}=3, P=0.001\right)$ showed that the risk of dying after release was positively related to the concentration of urea nitrogen and negatively related to hematocrit level and the al- bumin/globulins ratio (Table 2). The risk of dying was not significantly associated with sex, pregnancy at the start of quarantine, release area, or quarantine length. We saw similar findings when the regression model was fitted to data obtained up to 90 days after release, but no model fitted the data obtained between days 23 and 90 of the survey, showing that the association between the biochemical parameters and the risk of dying was observable only during the first days after release.

\section{DISCUSSION}

Capture and transport have been identified as main sources of stress for wild species (Jacobson et al. 1978, Brelurut et al. 1991, Millspaugh et al. 2000). Our results supported these findings and suggested that wild rabbits also experience stress at these times. The relatively high total bilirubin 
concentrations at the start of quarantine were due primarily to the high levels of unconjugated bilirubin that likely resulted secondarily from increased hemoglobin from hemolysis caused by acute stress (White et al. 1991, Vassart et al. 1992, Marco et al. 1997, Millspaugh et al. 2000). In previous studies with other species, an acute stress response was usually associated with high hematocrit values, since stress causes splenic contraction and release of erythrocytes to the peripheral circulation ( Jacobson et al. 1978, Marco et al. 1997). In our findings, however, blood collection took place some time after the initial stress of capture and hemolysis may explain lower hematocrit values observed at the start of quarantine relative to those at the end of quarantine.

Our finding that the AST and ALT levels observed at the start of quarantine were high but declined significantly throughout the quarantine period also suggested that capture and transport caused high stress in rabbits. Both enzymes were associated with muscle damage due to the stress of capture in wild species, with decreases observed a few days after capture (Jacobson et al. 1978; Brelurut et al. 1991; Marco et al. 1997, 1998). Similar results were observed in domestic rabbits after heat stress (Amici et al. 2000); however, determination of creatine kinase (CK) activity may be a more useful approach to determine muscle injury in future surveys.

Blood urea nitrogen may be significantly altered by protein catabolism, protein synthesis, or both (Fox and Laird 1999). Increased urea nitrogen was related to increased tissue catabolism as a stress response in wild and domestic species (Jacobson et al. 1978, Kock et al. 1987, Marco et al. 1998), including domestic rabbits (Amici et al. 2000). Increased urea nitrogen levels, however, were also associated with low protein intake (Morisse et al. 1982) or ingestion of low quality protein (i.e., when amino acids are not properly balanced; Bora et al. 2001). Hence, the decreased levels of urea nitrogen we observed in wild rabbits through the quarantine period may have been due to a decrease in the acute stress response after capture and transport and to the intake of high quality proteins in a diet based on commercial pellets (increasing their physiological condition). Our finding that urea nitrogen levels decreased sharply after 2 weeks of quarantine (i.e., when the rabbits were allowed full access to the commercial feed) is further support for the latter.

Although urea nitrogen levels are usually influenced by pregnancy or sex, and creatinine and total protein are usually influenced by pregnancy
(Fox and Laird 1999), we found no such statistical associations for any of these parameters, possibly because the stress response overlapped any baseline differences. Also, we did not observe any association between biochemical parameter and quarantine length, except for hematocrit and urea nitrogen that were only altered in the group of rabbits released after 2 weeks of quarantine, a group that had the lowest body mass ratio. This biochemical profile suggested that quarantine induced less stress than capture and transport. Thus, the pattern we observed in this set of biochemical parameters through quarantine may be due to the access to high quality feed beginning after 2 weeks of quarantine and to the gradual decrease of the acute stress response after capture and transport. Our lack of baseline reference values for rabbit biochemical parameters in the wild, however, precluded us from evaluating the degree of adaptation of these rabbits to restraint during quarantine.

Seroconversion induced by vaccination against myxomatosis and RHD was not affected by quarantine length. This was likely due to antibodies against both viruses reaching steady-state levels within 2 weeks of vaccination (Argüello 1986, 1991). While we observed no correlation between any biochemical parameter and seroconversion, our results suggested that the efficacy of vaccination, as shown by the proportion of initially seronegative rabbits that were seropositive at the end of quarantine, was lower than in domestic rabbits, especially for vaccination against RHD (Pages 1989, Argüello 1991, Smid et al. 1991, Simon et al. 1993). These findings suggested that the effects of booster vaccination during quarantine should be evaluated and the efficacy of immunization against both diseases improved in the future.

We found that the risk of dying through quarantine was associated with biochemical parameters of the rabbits at the start of quarantine. Since the physiological condition of the rabbits at their reception in the quarantine facilities was probably related to the severity of pre-bleeding events (i.e. capture, prequarantine restraining, transport), the biochemical parameters associated with mortality during quarantine likely reflected the acute stress response of individual rabbits to capture and handling (Calvete et al. 2004), as well as the length of the restraining period prior to quarantine. Thus, the association of risk of dying during quarantine with low serum protein levels and high conjugated/unconjugated bilirubin ratio (mainly due to high levels of conjugated bilirubin) may reflect the physiological deterioration that occurred between capture and re- 
ception at the quarantine facilities, since rabbits had no access to high quality food during this period.

The heterophil/lymphocyte ratio and the levels of AST and creatinine are usually associated with the alarm phase of the stress response. Catecholamines are responsible for the mobilization of heterophils during the first hours after the onset of a stressful situation, as well as for increased catabolism. In wild ungulates, AST has been associated with mortality by capture myopathy due to stress (Vassart et al. 1992), while in stressed bighorn sheep, creatinine, which is a subproduct of muscle catabolism, has been associated with short-term mortality (Kock et al. 1987). Moreover, Amici et al. (2000) showed that immune cell functions in rabbits may be strongly compromised by their exposure to acute stress, leading to possible reduction in the resistance to infectious agents. Hence, rabbit mortality during quarantine may be partially related to the intensity of acute stress due to capture and transport and to incorrect handling prior to quarantine (e.g., trappers not giving rabbits access to feed for long periods after capture).

Our finding that mortality due to translocation was higher during the first days after release is in agreement with previous findings (Calvete et al. 1997, Letty et al. 2002). While the risk of death after release was positively related to urea nitrogen levels and negatively to hematocrit and the albu$\mathrm{min} /$ globulin ratio, it was not dependent on the length of time the rabbits were kept in quarantine. This suggested that mortality was not dependent on the length of restraint, but it may be negatively associated with the rabbits' physiological condition at the time of release. The widespread variability of biochemical parameters and body mass we observed was indicative of the high variability of body condition among individual rabbits during the quarantine period. Since body condition was partially dependent on the unlimited access of rabbits to high quality feed, those rabbits released after 2 weeks of quarantine may have been in poorer overall condition; thus, they had a lower chance of survival after release.

\section{MANAGEMENT IMPLICATIONS}

The most common procedure for rabbit translocations is the simultaneous movement of several tens or hundreds of individuals (Calvete et al. 1997) and the use of quarantine facilities designed to control and manage high numbersof individuals due to small body size and low individual economic cost. These facilities increase the degree of confinement of animals and decrease the quality of environment during quarantine, and they may affect behavior and reduce the fitness and survival of animals after release, especially after long quarantines. However, our results showed that survival was not associated with quarantine length and suggested that the practice of quarantine in small mammal translocations using this type of facilities would be feasible.

We suggest the following improvements in future rabbit translocation programs that include quarantine: (1) Decrease the acute stress induced by capture, transport, and inappropriate handling prior to quarantine (e.g., by decreasing elapsed time between capture and the start of quarantine). (2) Improve the efficacy of current vaccination protocols against myxomatosis and RHD (e.g., by testing alternative immunization schemes that include booster injections). (3) Facilitate more rapid access to high quality feed to reduce the initial deterioration of body condition. (4) Measure variations in body condition throughout quarantine (e.g., body mass). Thus, the release from quarantine of an individual rabbit will also be determined by its physical condition, not merely by elapsed time in quarantine.

\section{ACKNOWLEDGMENTS}

The Local Government of Extremadura and Doñana National Park (Organismo Autónomo de Parques Nacionales) provided partial funding. A postdoctoral fellowship to C. Calvete was funded by the High Council of Scientific Research (CSIC) (REN2001-0448/GLO and PBI-02-004). E. Angulo was supported by a grant from the Spanish Ministry of Science and Technology (FPI-MIT). We thank S. Cabezas Ruiz for useful discussions.

\section{LITERATURE CITED}

Amici, A., O. France, P. Mastrodiacono, N. Merendino, M. NARDini, AND G. TOMASSI. 2000. Short term acute heat stress in rabbits: functional, metabolic and immunological effects. World Rabbit Science 3:11-16.

Angulo, E. 2003. Factores que afectan a la distribución y abundancia del conejo en Andalucía. Dissertation, Complutense University, Madrid, Spain. [In Spanish.]

—, AND B. COOKE. 2002. First synthesize new viruses then regulate their release? The case of the wild rabbit. Molecular Ecology 11:2703-2709.

Argüello, J. L. 1986. Contribución a la profilaxis de la mixomatosis del conejo mediante el uso de una cepa homóloga. Medicina Veterinaria 3:91-103. [In Spanish.]

- 1991. La enfermedad hemorrágica viral del conejo: vacunación y respuesta inmunológica. Revue Scientifique et Technique Office International des Epizooties 10:459-467. [In Spanish.]

- , A. Llanos, And L. I. Perez. 1988. Enfermedad Hemorrágica del conejo en España. Medicina Veterinaria 5:645-650. [In Spanish.]

Bora, A., R. N. Baruah, K. K. Baruah, and R. K. Bora. 2001. Influence of different concentrate: roughage ra- 
tio on certain blood constituents of rabbits. Indian Veterinary Journal 78:1044-1045.

Branco, M. 1995. Contribuçao para o estudo da genética bioquímica e populacional do coelho, Oryctolagus $\mathrm{cu}$ niculus. Dissertation, Universidade do Porto, Douro Litoral, Portugal. [In Portuguese.]

Brelurut, A., M. Auguergouz, And M. Verdier. 1991. Effets de la capture et du transport sur quelques constantes sanguines du jeune cerf (Cervus elaphus). Gibier Faune Sauvage 8:271-282.

Calvete, C., AND R. Estrada. 2004. Short-term survival and dispersal of translocated European wild rabbits. Improving the release protocol. Biological Conservation 120:507-516.

—, - J. J. OsÁcAR, J. LuCIENTES, AND R. VIllaFUERTE 2004. Short-term negative effects of vaccination campaigns against Myxomatosis and Viral Haemorrhagic Disease (VHD) on the survival of European wild rabbits. Journal of Wildlife Management 68:198-205.

- - R. Rillafuerte, J. Lucientes, and J. J. OsÁCAR. 2002. Epidemiology of viral hemorrhagic disease (VHD) and myxomatosis in the wild rabbit (Oryctolagus cuniculus) in the mid-Ebro valley, Spain. Veterinary Record 150:776-782.

—, R. Villafuerte, J. Lucientes, and J. J. Osácar. 1997. Effectiveness of traditional wild rabbit restocking in Spain. Journal of Zoology 241:271-277.

Delibes, M., AND F. Hiraldo. 1981. The rabbit as prey in the Mediterranean ecosystem. Pages 614-622 in K. Myers, and C.D. MacInnes, editors. Proceedings of the world lagomorphs conference. University of Guelph, Ontario, Canada.

FERRAND, N. 1995. Variaçao genética de proteínas em populaçoes de coelho (Oryctolagus cuniculus). Dissertation, Universidade do Porto, Douro Litoral, Portugal. [In Portuguese.]

Fox, R. R., AND C. W. Laird. 1999. The rabbit. Pages 71-84 in W. F. Loeb and F. W. Quimby, editors. The clinical chemistry of laboratory animals. Quimby, Miami, Florida, USA.

Griffith, B., M. Scott, J. W. Carpenter, And C. Reed. 1989. Translocation as a species conservation tool: status and strategy. Science 245:477-480.

IUCN 1996. IUCN/SSC guidelines for re-introductions. 41st Meeting of the IUCN Council, Gland, Switzerland.

JACOBSON, E. R. 1993. Implications of infectious diseases for captive propagation and introduction programs of threatened/endangered reptiles. Journal of Zoo and Wildlife Medicine 24:245-255.

JACOBSON, H. A., R. L. KirKPATRICK, H. E. BuRKhaRT, AND J. W. DAVIs. 1978. Hematologic comparisons of shot and live trapped cottontail rabbits. Journal of Wildlife Diseases 14:82-88.

Kock, M. D., R. K. Clarck, C. E. Franti, D. A. Jessup, and J. D. Wehausen. 1987. Effects of capture on biological parameters in free-ranging bighorn sheep (Ovis canadiensis): evaluation of normal, stressed and mortality outcomes and documentation of postcapture survival. Journal of Wildlife Diseases 23:652-662.

Letty, J., S. Marchandeau, F. Reitz, J. Clobert, and F. SARRAZIN. 2002. Survival and movements of translocated wild rabbits (Oryctolagus cuniculus). Game and Wildlife Science 19:1-23.

Marco, I., L. Viñas, R. Velarde, J. PAStor, and S. LaVin. 1997. Effects of capture and transport on blood para- meters in free-ranging mouflon (Ovis ammon). Journal of Zoo and Wildlife Medicine 28:28-433.

stress response to repeated capture in Mouflon (Ovis ammon): physiological, haematological and biochemical parameters. Journal of Veterinary Medicine A 45:243-253.

Millspaugh, J. J., M. A. Coleman, P. J. Bauman, and K. J. RAEDEKE. 2000. Serum profiles of American elk, Cervus elaphus, at the time of handling for three capture methods. Canadian Field-Naturalist 114:196-200.

Morisse, J. P., J. P. CotTe, AND D. Huonnic. 1982. Influence du circuit d'approvisionnement sur le comportement sanitaire du veau. Modifications hématologiques et biochimiques chez le Veau stressé. Recueil Médecine Vétérinaire 158:307-314. [In French.]

MuÑoz, G. 1960. Anverso y reverso de la mixomatosis. Dirección General de Montes, Caza y Pesca Fluvial. Madrid, Spain. [In Spanish.]

NAyerul, M., AND T. R. Smith. 1996. Reintroduction of arabian sand gazelle Gazella subgutturosa marica in Saudi Arabia. Biological Conservation 76:203-207.

Pages, A. 1989. Consideraciones técnicas de la sueroterapia y de la profilaxis vacunal en la enfermedad hemorrágica vírica del conejo (RHDV). Medicina Veterinaria 6:285-291. [In Spanish.]

Smid, B., L. VAliceK, L. RodaK, J. StepaneK, and E. JuRaK. 1991. Rabbit haemorrhagic disease: an investigation of some properties of the virus and evaluation of an inactivated vaccine. Veterinary Microbiology 26:77-85.

Simón, M. C., O. Gironés, J. L. Alonso, J. L. Múzquiz, J. García, C. Ortega, and R. Muguruza. 1993. Enfermedad vírica hemorrágica en el conejo industrial: eficacia de una vacuna inactivada en la protección frente a la inoculación experimental. Medicina Veterinaria 10:44-48. [In Spanish.]

SPALDing, M. G., AND D. J. ForRester. 1993. Disease monitoring of free-ranging and released wildlife. Journal of Zoo and Wildlife Medicine 24:271-280.

Therneau, T. M., AND P. M. Grambsch. 2001. Modeling survival data: extending the Cox Model. Second edition. Springer-Verlag, New York, USA.

Vassart, M. A., S. Greth, S. Anagariyah, and F. Mollet. 1992. Biochemical parameters following capture myopathy in one Arabian Oryx (Oryx leucoryx). Journal of Veterinary Medicine Science 54:1233-1235.

White, P. J., T. J. Kreeger, U. S. Seal, and J. R. Tester. 1991. Pathological responses of red foxes to capture in box traps. Journal of Wildlife Managament 55:75-80.

Viggers, K.L., D. B. Lindenmayer, and D. M. Spratt. 1993. The importance of disease in reintroduction programmes. Wildlife Research 20:687-698.

Wolf, C. M., B. Griffith, C. Reed, and S. A. Temple. 1996. Avian and mammalian translocations: update and reanalysis of 1987 survey data. Conservation Biology 10:1142-1154.

WoOdFord, M. H., AND R. A. KocK. 1991. Veterinary considerations in re-introduction and translocation projects. Symposium Zoological Society of London 62:101-110.

- AND P. B. Rossiter. 1993. Disease risks associated with wildlife translocation projects. Revue Scientifique et Technique Office International des Epizooties 12:115-135.

Associate Editor: Martin. 